\title{
Impact of an alien wavelength on wavelength division multiplexing transmission quality
}

\author{
Krzysztof Perlicki ${ }^{*}$ \\ Institute of Telecommunications, Warsaw University of Technology, Nowowiejska 15/19, 00-665 Warszawa
}

Received July 31, 2012; accepted September 19, 2012; published September 30, 2012

\begin{abstract}
The modern Dense Wavelength Division Multiplexing management system has no a priori knowledge of alien wavelength parameters. The result of this is unforeseeable impact of alien wavelength parameters on friendly wavelengths quality. We present a numerical simulation of the impact of an alien wavelength bandwidth on optical transmission quality. The obtained results indicate that an alien wavelength bandwidth must be restricted.
\end{abstract}

Optical Dense Wavelength Division Multiplexing (DWDM) transmission systems have been evolving from closed systems to open systems, where the optical layer is designed in a way that allows new transmitters and receivers to be connected to it without requiring any changes in the physical layer or planning and management tools that are needed to operate it. This trend is reflected in solutions for an alien wavelength [1-2].

The traditional DWDM system contains transponders which convert the signals suitable for colored (DWDM) light paths. The alien wavelength solutions are based on a colored interface which resides in the client equipment (e.g. IP router) [3]. This potentially saves transmission system costs, increases provisioning speed and allows greater transparency. However, the DWDM management system has no a priori knowledge of alien wavelength signal parameters (wavelength, bandwidth) [3].

The result of this is unforeseeable impact of alien wavelength parameters on friendly wavelengths quality. The wavelengths which are managed by the DWDM system are called friendly wavelengths [4]. The ability of DWDM networks to carry alien wavelengths with new and advanced formats (e.g. DPSK - Differential Phase Shift Keying) and bit rates has been demonstrated in a context of $40 \mathrm{~Gb} / \mathrm{s}$ alien wavelengths over DWDM networks that were designed for $10 \mathrm{~Gb} / \mathrm{s}$ signals [5]. Today, we have to focus on $40 \mathrm{~Gb} / \mathrm{s}$ or $100 \mathrm{~Gb} / \mathrm{s}$ alien wavelengths over DWDM systems that were designed for $40 \mathrm{~Gb} / \mathrm{s}$ signals.

In this paper, we present a numerical simulation of the impact of an alien wavelength bandwidth on DWDM channels crosstalk.

The crosstalk between an alien wavelength and DWDM

*E-mail: perlicki@ tele.pw.edu.pl friendly wavelengths is calculated.

The simulation setup is focused on an optical transmission system with a $40 \mathrm{~Gb} / \mathrm{s}$ or $100 \mathrm{~Gb} / \mathrm{s}$ alien wavelength and $40 \mathrm{~Gb} / \mathrm{s}$ friendly wavelengths. Besides, we take into account an alien wavelength that is added to the DWDM system by a multiplexer or an optical splitter (combiner).

The simulation setup is shown in Fig. 1. The presented DWDM system consists of four channels. The DWDM multiplexer and demultiplexer consist of optical super Gaussian filters. The super Gaussian filters of second order are considered. Considering a ITU-T channel spacing 50GHz $(194.05 \mathrm{THz}, 194.10 \mathrm{THz}, 194.15 \mathrm{THz}$ and 194.20THz) [6], the $3 \mathrm{~dB}$ bandwidths of multiplexer and demultiplexer filters are varied in a region between $10 \mathrm{GHz}$ and $60 \mathrm{GHz}$ for investigated modulation format and bit rates. Here, only optical crosstalk between an alien wavelength and friendly wavelengths is taken into account. The other effects (optical losses, chromatic dispersion, polarization dispersion or nonlinear effects) are neglected. The optical crosstalk (i.e. difference between proper signal and crosstalk signal) is calculated at demultiplexer port No. 3 (Figs. 1-2). The NonReturn to Zero Two-Phase Differential Phase Shift Keying (NRZ 2DPSK) modulation format at $40 \mathrm{~Gb} / \mathrm{s}$ and $100 \mathrm{~Gb} / \mathrm{s}$ channels was chosen for our investigation. Numerical simulations are realized with OptSim4.0 (RSoft Inc.).

The following four cases are considered:

- Case 1: The 40Gb/s alien wavelength (Tx2) and 40Gb/s friendly wavelengths ( $\mathrm{Tx} 1, \mathrm{~T} \times 3, \mathrm{~T} \times 4)$. The friendly wavelengths and an alien wavelength are combined into an optical fiber by means of a multiplexer (Fig. 1).

- Case 2: The 100Gb/s alien wavelength (Tx2) and $40 \mathrm{~Gb} / \mathrm{s}$ friendly wavelengths (Tx1, Tx3, Tx4). The friendly wavelengths and an alien wavelength are combined into an optical fiber by means of a multiplexer (Fig. 1).

- Case 3: The 40Gb/s alien wavelength (Tx2) and 40Gb/s friendly wavelengths ( $\mathrm{Tx} 1, \mathrm{~T} \times 3, \mathrm{~T} \times 4)$. The friendly wavelengths are combined into an optical fiber by means of a multiplexer. In contrast, an alien wavelength is added to the DWDM system via an 
optical splitter (Fig. 2).

- Case 4: The 100Gb/s alien wavelength (Tx2) and $40 \mathrm{~Gb} / \mathrm{s}$ friendly wavelengths (Tx1, Tx3, Tx4). The friendly wavelengths are combined into an optical fiber by means of a multiplexer. In contrast, an alien wavelength is added to the DWDM system via an optical splitter (Fig. 2).

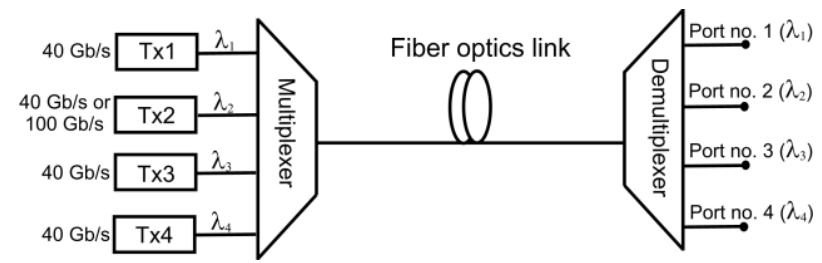

Fig. 1. Diagram of the simulated DWDM system with an alien wavelength which is added to the DWDM system via a multiplexer;

$\mathrm{Tx} 1, \mathrm{Tx} 2, \mathrm{~T} \times 4-$ friendly wavelength transmitters, Tx 2 - alien wavelength transmitter, $\lambda_{1}, \lambda_{3}, \lambda_{4}-$ friendly wavelengths; $\lambda_{2}-$ alien wavelength.

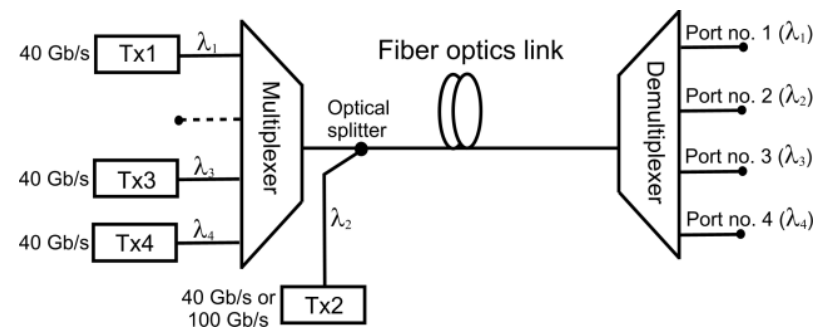

Fig. 2. Diagram of the simulated DWDM system with an alien wavelength which is added to the DWDM system via an optical splitter;

Tx1, Tx2, Tx4 - friendly wavelength transmitters, Tx 2 -alien wavelength transmitter, $\lambda_{1}, \lambda_{3}, \lambda_{4}-$ friendly wavelengths; $\lambda_{2}-$ alien wavelength.

Figures 3, 4, 5 and 6 show the crosstalk values for Case $1,2,3$ and 4 , respectively.

(a)

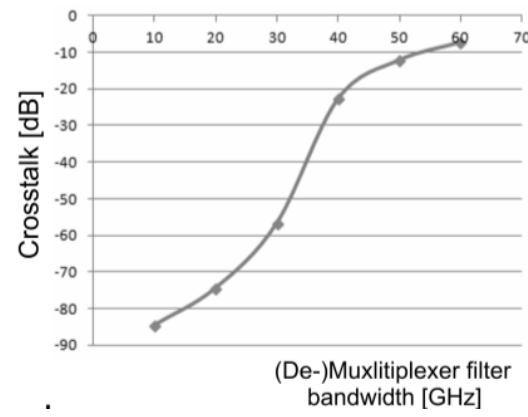

(b)

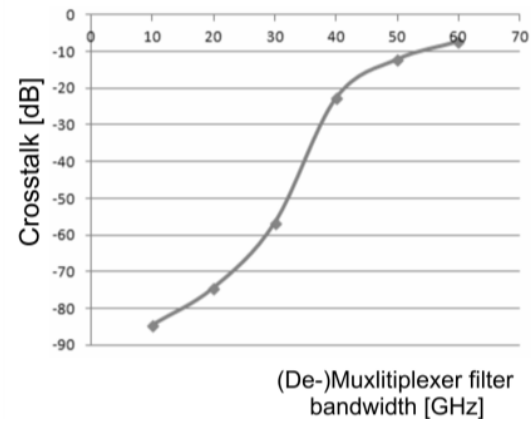

Fig. 3. Crosstalk values versus demultiplexer and multiplexer filter bandwidth in Case 1 (a) and in Case 2 (b).

For Case 1 and 2, narrow band filtering (10GHz $40 \mathrm{GHz})$ is required both at a multiplexer and demultiplexer filter in order to achieve a proper crosstalk value. The proper transmission quality is achieved for crosstalk values less than $-20 \mathrm{~dB}$ [7].

Through narrow band filtering a better separation of alien and friendly wavelengths can be realized and the overlap between adjacent DWDM channels is reduced. In turn, the alien wavelength is not filtered by a multiplexer filter in Cases 3 and 4.

(a)

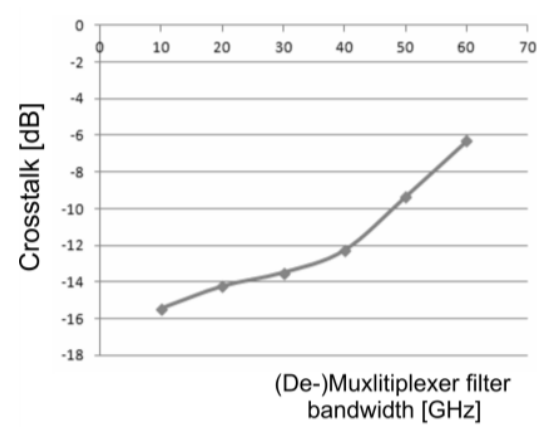

(b)

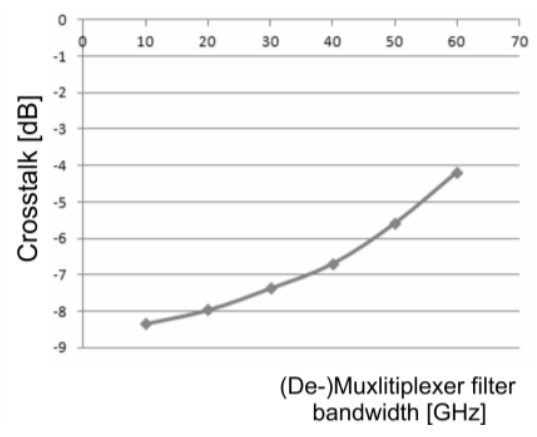

Fig. 4. Crosstalk values versus demultiplexer and multiplexer filter bandwidth in Case 3 (a) and in Case 4 (b). 
The result of this is large crosstalk values (greater than $-20 \mathrm{~dB}$ ). These crosstalk values are inappropriate for telecommunication applications.

In conclusion, the obtained results indicate that unfiltered (or improperly filtered) $40 \mathrm{~Gb} / \mathrm{s}$ or $100 \mathrm{~Gb} / \mathrm{s}$ alien wavelengths induce large crosstalk. The alien wavelength bandwidth must be restricted by a multiplexer filter or a dedicated optical filter. The narrow band filtering (bandwidth $\leq 40 \mathrm{GHz}$ ) both at a multiplexer and a demultiplexer is essential required in order to achieve a proper crosstalk value for modern $40 \mathrm{~Gb} / \mathrm{s}$ and $100 \mathrm{~Gb} / \mathrm{s}$ DWDM systems.

\section{References}

[1] O. Gerstel, R. Cassata, L. Paraschis, Conference on Optical Fiber Communication/National Fiber Optic Engineers Conference, NthF1, San Diego, USA (2009).

[2] S. Melle, G. Bennett, Ch. Liou, Conference on Optical Fiber Communication/National Fiber Optic Engineers Conference, NthF2, San Diego, USA (2009).

[3] A. Lord, Y.R. Zhou, P. Wright, European Conference on Optical Communications, Tu.6.K.2, Geneva, Switzerland (2011).

[4] R. Kunze, A framework for Management and Control of optical interfaces supporting G.698.2, Internet Engineering Task Force Internet-Draft (2011).

[5] D. Ventorini, E. Moura, L. Paraschis, Conference on Optical Fiber Communication/National Fiber Optic Engineers Conference, NME3, San Diego, USA (2008).

[6] ITU-T Recommendation G.692 (1998).

[7] G.P. Agrawal, Fiber-Optic Communication Systems, (New York, John Wiley \& Sons, 2002). 